Copyright (C) 2013 IEEE. Personal use of this material is permitted. Permission from IEEE must be obtained for all other uses, in any current or future media, including reprinting/republishing this material for advertising or promotional purposes, creating new collective works, for resale or redistribution to servers or lists, or reuse of any copyrighted component of this work in other works. 


\section{A Frequency Domain Equalizer for Amplify-and-Forward Underwater Acoustic Relay Communication Systems}

\author{
Khoa Xuan Nguyen, Yue Rong \\ Dept. of Electrical and Computer Engineering \\ Curtin University of Technology, WA, Australia \\ Email: xuan.khoa.nguyen@curtin.edu.au \\ y.rong@curtin.edu.au
}

\author{
Zhiqiang He \\ School of Information and Communication Engineering \\ Beijing University of Posts and Telecommunications \\ Beijing, China \\ Email: hezq@bupt.edu.cn
}

\begin{abstract}
In this paper, we apply the amplify-and-forward relay technique to simultaneously increase the range and data rate of underwater acoustic communication by dividing the channel between transmitter and receiver into two hops. Due to the application of the relay node, the delay spread of the effective transmitter-relay-receiver multipath channel is longer than that of the direct transmitter-receiver channel, which increases the complexity of channel equalization at the receiver. To reduce the computational complexity of channel equalization, a fractionally-spaced frequency domain equalizer (FS-FDE) is designed in this paper. Simulation results illustrate that compared with the direct path communication, significant bit-error-rate performance improvement can be achieved through using relay technique in underwater acoustic communication.
\end{abstract}

Index Terms-Underwater acoustic communication, amplifyand-forward relay, equalization.

\section{INTRODUCTION}

Digital communication through underwater acoustic (UA) channels is very challenging due to severe signal degradation caused by harsh underwater channels, where the absorption loss of acoustic wave increases with distance and the carrier frequency. Signals transmitted through UA channels whose characteristics vary with time are also subject to severe fading and strong multipath interference.

Multipath reflections depend on the channel geometry and the frequency of transmitted signals. Thus, acoustic wave propagation in shallow water is much different from the one in deep water. In fact, the intersymbol interference (ISI) span of the shallow water UA channel is longer than that of the deep water channel. In addition, the absorption loss of acoustic wave increases with distance and frequency, resulting in reduction of available bandwidth in long range communication [1]. For example, the bandwidth available for UA communication over $10 \mathrm{~km}$ is $10 \mathrm{kHz}$, and it reduces to $1 \mathrm{kHz}$ for a transmission range of $100 \mathrm{~km}$.

To address these difficulties, recent researches focus on techniques such as signal design and transceiver structure design which are two main approaches received most of interests. The focus of signal design methods is to improve the system bandwidth efficiency through, for example, phase- coherent modulation/detection methods. Systems implemented based on these methods can achieve up to $2 \mathrm{kbps}$ over long range channels and up to $40 \mathrm{kbps}$ over shallow water mediumrange channels [2].

On the other hand, transceiver structure design approaches such as array processing [3] and cooperative communication [4]- [6] have not received much attention until recently, particularly with cooperative communication. By relaying signals at intermediate terminals between source and destination, cooperative communication technique improves total network channel capacities as well as reduces required transmission power. Cooperative communication scheme also exploits diversity characteristics of channels by combining signals propagated through different hops.

In this paper, we investigate a cooperative UA communication system consisting of a source, a destination, and a relay, and compare its performance with the direct source-destination communication system. Due to the application of the relay node, the delay spread of the effective transmitter-relayreceiver multipath channel is longer than that of the direct transmitter-receiver channel, which increases the complexity of channel equalization at the receiver. To reduce the computational complexity of channel equalization, a fractionallyspaced frequency domain equalizer (FS-FDE) [7] is designed in this paper.

We study the bit-error-rate (BER) performance of a single relay two-hop cooperative UA communication system with a fractionally-spaced linear minimum mean-squared error frequency domain equalizer (LMMSE-FDE). Numerical simulations are carried out with real at-sea channel measurement data, and different over-sampling factors are used for performance comparison. Simulation results show that compared with the direct path communication, significant BER performance improvement can be achieved through using relay technique in UA communication.

This paper is organized as follows. Section II introduces the signal model and the single relay cooperative communication scheme. Section III describes the fractionally-spaced LMMSEFDE. Simulation results are shown and discussed in Section 
IV. Section V presents the conclusions. Throughout this paper, scalar quantities are denoted with lower or upper case normal letters, vectors are denoted with bold-faced lower case letters, and matrices are denoted with bold-faced upper case letters. Superscripts "*", " $T$ ", " $H$ " and " -1 " denote complex conjugate, transpose, conjugate transpose and inverse, respectively.

\section{System Model}

In this paper, a simplest two-hop relay network with three nodes is considered, where node $\mathrm{S}$ (source) sends information to node $\mathrm{D}$ (destination) with the aid of node $\mathrm{R}$ (relay) as shown in Fig. 1. We denote $\mathbf{h}_{s r} \triangleq\left[h_{s r}(0), \cdots, h_{s r}\left(L_{s r}-1\right)\right]^{T}$, $\mathbf{h}_{s d} \triangleq\left[h_{s d}(0), \cdots, h_{s d}\left(L_{s d}-1\right)\right]^{T}$, and $\mathbf{h}_{r d} \triangleq$ $\left[h_{r d}(0), \cdots, h_{r d}\left(L_{r d}-1\right)\right]^{T}$ as the source-relay, sourcedestination and relay-destination channel multipath profiles with length of $L_{s r}, L_{s d}$ and $L_{r d}$ respectively. The transmission is conducted in two hops: first hop from source $\mathrm{S}$ to both relay $\mathrm{R}$ and destination $\mathrm{D}$, second hop from relay $\mathrm{R}$ to destination D. At the relay, received signals from source are amplified before being forwarded to destination. The amplification coefficient is determined based on the transmission power constraint at the relay. We assume that source and relay have the same transmission power.

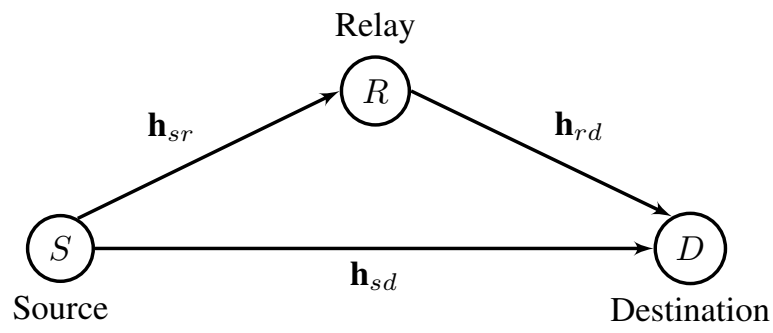

Fig. 1: A three-node two-hop communication system.

Fig. 2 illustrates the system block diagram. Signals are transmitted from source to destination via two different channels: direct path via source-destination channel $h_{s d}(i), i=$ $0,1, \ldots, L_{s d}-1$ and relayed path via source-relay, $h_{s r}(i), i=$ $0,1, \ldots, L_{s r}-1$, and relay-destination channels $h_{r d}(i), i=$ $0,1, \ldots, L_{r d}-1 \cdot v_{d}(n)$ and $v_{r}(n)$ are additive noises at the destination and relay, respectively. Furthermore, $A_{r}$ denotes the coefficient of the amplify-and-forward relay.

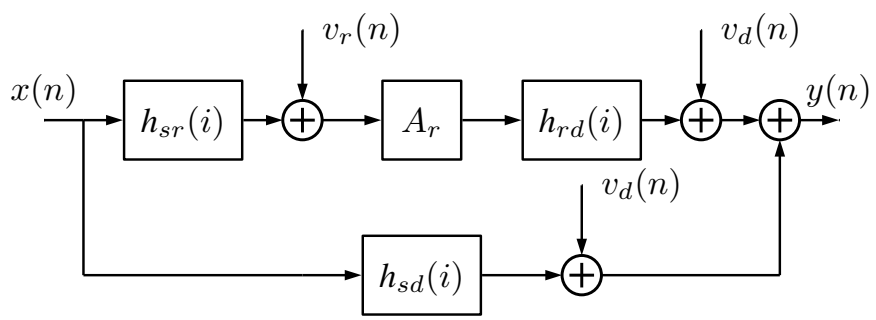

Fig. 2: System block diagram.

In the first hop, a block of $N$ symbols $x(n), n=$ $0,1, \ldots, N-1$ is transmitted from source to relay via $h_{s r}(i)$ channel and to destination via $h_{s d}(i)$ channel. The signals received at relay $y_{s r}(n)$ and destination $y_{s d}(n)$ can be written as

$$
\begin{aligned}
& y_{s r}(n)=\sqrt{P_{o}} \sum_{i=0}^{L_{s r}-1} h_{s r}(i) x(n-i)+v_{r}(n) \\
& y_{s d}(n)=\sqrt{P_{o}} \sum_{i=0}^{L_{s d}-1} h_{s d}(i) x(n-i)+v_{d}(n)
\end{aligned}
$$

where $P_{o}$ is the transmission power of the source. In the second hop, the received signal block from source is amplified with coefficient $A_{r}$ before being forwarded to destination. The received signal at destination in the second hop can be modeled as

$$
y_{r d}(n)=A_{r} \sum_{i=0}^{L_{r d}-1} h_{r d}(i) y_{s r}(n-i)+v_{d}(n)
$$

where the amplification gain $A_{r}$ of the relay is calculated as

$$
A_{r}=\sqrt{\frac{P_{r}}{P_{o} \sum_{i=0}^{L_{s r}-1} h_{s r}(i) h_{s r}^{*}(i)}}
$$

where $P_{r}$ is the transmission power of the relay.

The overall received signals at the destination are the combination of signals through the direct source-destination channel $y_{s d}$, and signals through the relay $y_{r d}$ as shown in Fig. 2, which can be written as

$$
y(n)=\sum_{i=0}^{L-1} h(i) x(n-i)+v(n)
$$

where

$$
h(k)=A_{r} \sum_{i=0}^{L-1} h_{r d}(i) h_{s r}(k-i)+h_{s d}(k)
$$

is the equivalent complex channel between source and destination, and

$$
v(n)=v_{d}(n)+A_{r} \sum_{i=0}^{L_{r d}-1} h_{r d}(i) v_{r}(n-i)
$$

is the equivalent additive noise at the destination.

\section{Fractionally-SPACED FREQUENCY Domain LINEAR EQUALIZATION}

The transmitter and receiver structures are illustrated in Fig. 3. At the transmitter, modulated symbols are divided into blocks with length of $\mathrm{N} / \mathrm{m}$, where $m$ is the up-sampling factor. Each block is sampled at the rate of $m / T$, where $T$ is the symbol period, before being appended with a cyclic prefix of length $L$. The cyclic prefix for each block is the repetition of last $L$ symbols of the block to form a transmitted data block [8]. The transmitted signal is distorted by the equivalent channel $h(i)$ and corrupted by additive noise $v(n)$ as in (5). The received signal is then passed through receiver filter $g$ before removing the cyclic prefix. Each block of received signal after removing cyclic prefix can be written as

$$
\mathbf{r}=\mathbf{H} \mathbf{x}+\mathbf{v}
$$




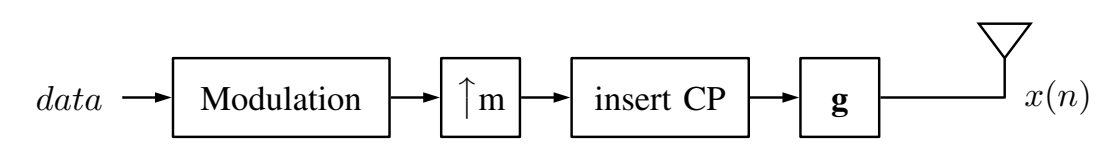

(a) Transmitter model.

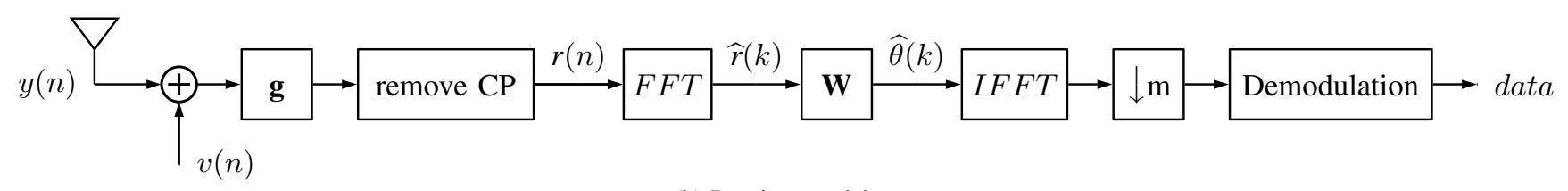

(b) Receiver model.

Fig. 3: Transceiver structure.

where $\mathbf{r}=[r(0), \ldots, r(N-1)]^{T}, \mathbf{x}=[x(0), \ldots, x(N-1)]^{T}$ and $\mathbf{v}=[v(0), \ldots, v(N-1)]^{T}, \mathbf{H}$ is a circulant matrix with first column equal to the impulse response of the equivalent channel in (6), and it has the eigenvalue decomposition of

$$
\mathbf{H}=\mathbf{F}^{H} \boldsymbol{\Lambda} \mathbf{F}
$$

where $\mathbf{F}(i, j)=1 / \sqrt{N} e^{-j 2 \pi i j / N}, 0 \leq i, j \leq N-1$ is the normalized discrete Fourier transform (DFT) matrix and $\boldsymbol{\Lambda}$ is a diagonal matrix whose $(k, k)$-th element is $\lambda_{k}=$ $\sum_{i=0}^{L-1} h(i) e^{-j 2 \pi k i / N}$. The signal vector $\mathbf{r}$ is then transferred to the frequency domain as

$$
\begin{aligned}
\widehat{\mathbf{r}} & =\mathbf{F r} \\
& =\mathbf{F} \mathbf{F}^{H} \boldsymbol{\Lambda} \mathbf{F x}+\mathbf{F} \mathbf{v} \\
& =\boldsymbol{\Lambda} \widehat{\mathbf{x}}+\widehat{\mathbf{v}}
\end{aligned}
$$

where $\widehat{\mathbf{x}} \triangleq \mathbf{F x}$ and $\widehat{\mathbf{v}} \triangleq \mathbf{F v}$ are the signal vector and noise vector in the frequency domain, respectively.

The $N$-point DFT output $\widehat{\mathbf{r}}$ is processing by a linear filter as

$$
\widehat{\boldsymbol{\theta}}=\mathbf{W} \widehat{\mathbf{r}}
$$

where the weight matrix $\mathbf{W}$ can be obtained from the minimum mean square error (MMSE) criterion [7] as

$$
\mathbf{W}=\left(\boldsymbol{\Lambda}^{H} \boldsymbol{\Omega}^{-1} \boldsymbol{\Lambda}+\frac{1}{P_{o}} \mathbf{I}\right)^{-1} \boldsymbol{\Lambda}^{H} \boldsymbol{\Omega}^{-1} .
$$

Here I stands for an identity matrix, $\boldsymbol{\Omega}=\mathbb{E}\left\{\widehat{\mathbf{v}}^{H}\right\}$ is the correlation matrix of received noise samples with the $(i, j)$-th element given by $\Omega(i, j)=1 / N \sum_{k=0}^{N-1} \widehat{v}(k) \widehat{v}^{*}(k-i+j)$. The equalized symbols are then transferred back to time domain through inverse DFT before being decimated with the factor of $m$.

\section{Simulation Results}

The system is setup with a source-relay distance of 498 meters and a source-destination distance of 935 meters at depth of 15 meters. The delay power spectrum for the sourcerelay channel is plotted in Fig. 4 and the source-destination channel's profile is shown in Fig. 5. The channel profile of the relay-destination hop is similar to that of the source-relay hop. The channel measurement was conducted in April 2012 in the Indian Ocean off Cottesloe, Western Australia in shallow water.

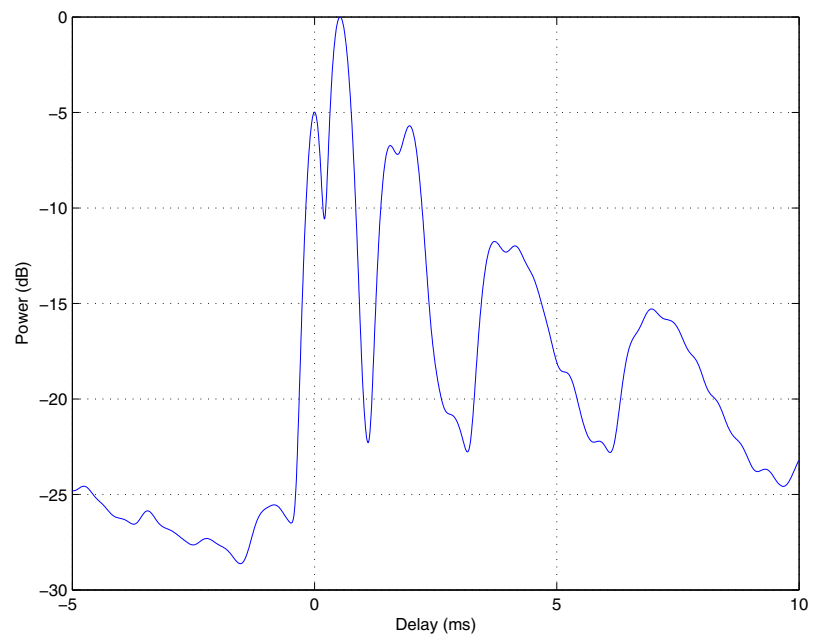

Fig. 4: Delay power spectrum for $498 \mathrm{~m}$ transmission at 337 degree heading.

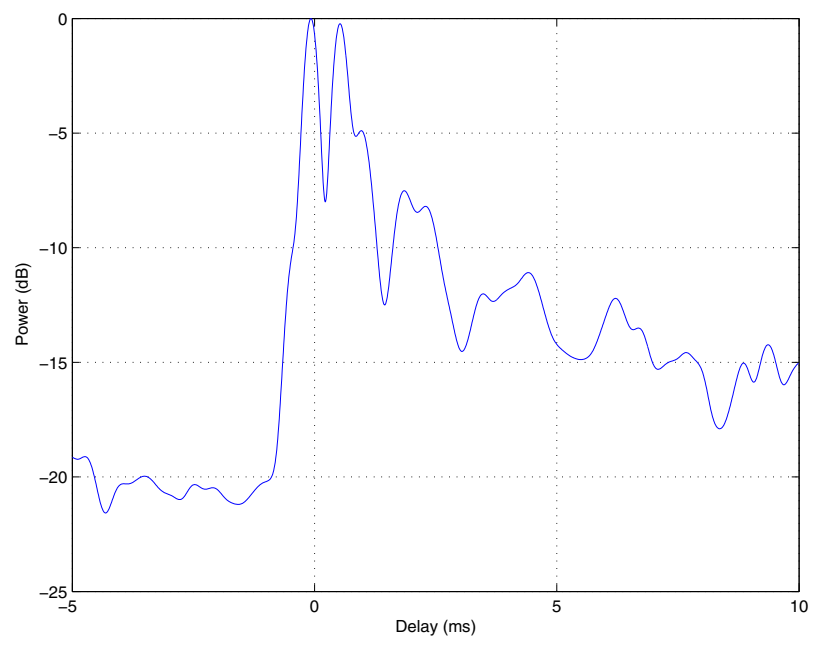

Fig. 5: Delay power spectrum for $935 \mathrm{~m}$ transmission at 337 degree heading. 
Equivalent channel $h$ in (6) has the delay power spectrum plotted in Fig. 6. Both source and relay transmit with unit power. Modulation method is QPSK and square-root raisedcosine filter with roll-off factor of 0.22 is used. The 1024-point FFT and IFFT are used for the FDE. The carrier frequency is $12 \mathrm{kHz}$ and the data rate is $5 \mathrm{kHz}$.

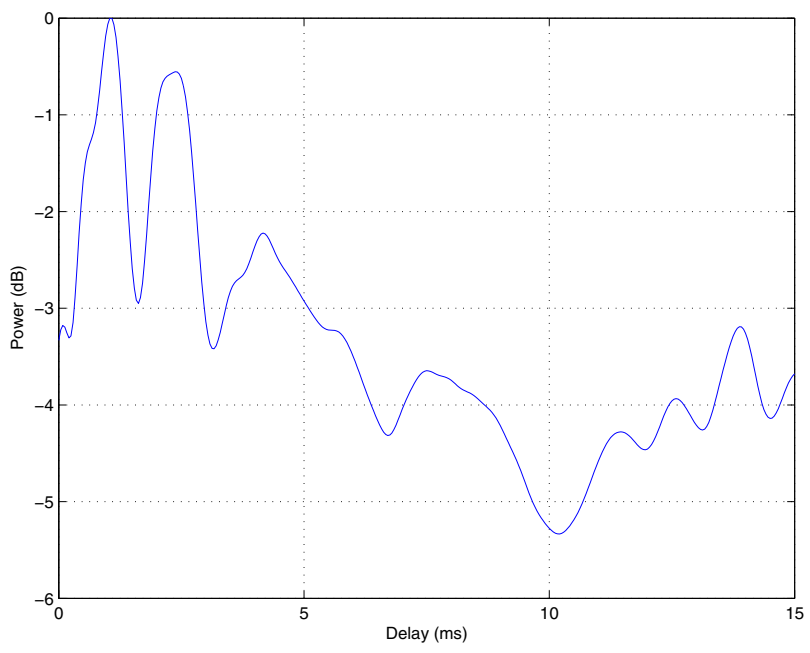

Fig. 6: Delay power spectrum for equivalent channel with relay.

The simulated BER performance of communication through relayed channel versus direct path communication is plotted in Fig. 7. For comparison, the performance of theoretical AWGN channel is also plotted. For a fair comparison, in the direct communication system, the source transmits with twice the power of that in the relayed transmission case. It can be clearly seen from Fig. 7 that relay-aided transmission performs better than direct path communication. In particular at a BER of $10^{-3}$, it gains about $2 \mathrm{~dB}$ in SNR with $m=8$.

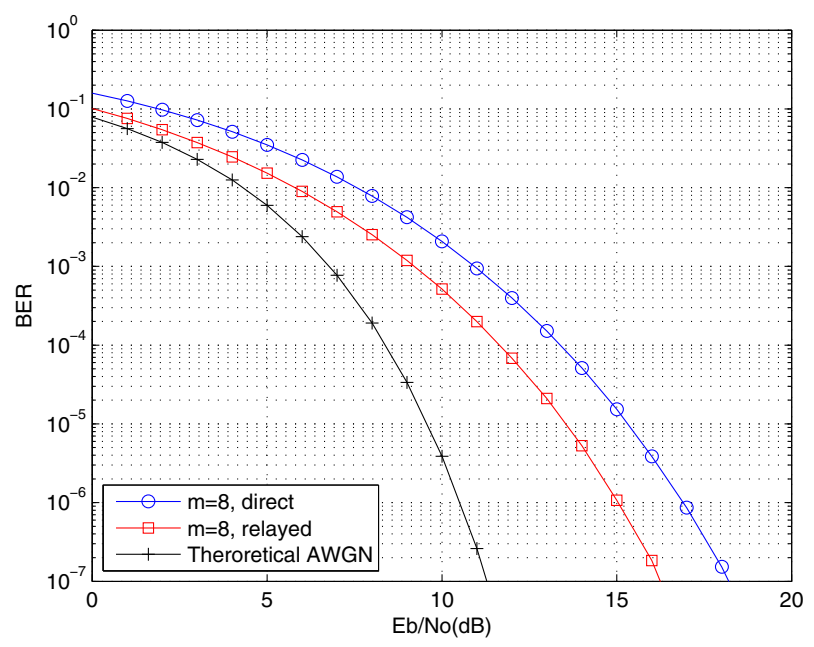

Fig. 7: Performance of relay-aided UA communication versus direct path communication.

The performance of fractionally-spaced FDE in relay-aided channel is plotted in Fig. 8. Symbol-spaced equalizer with $m=2$ and fractionally-spaced equalizers with $m=4$ and $m=8$ are simulated. It can be clearly seen that fractionallyspaced FDE significantly improves the BER performance compared with the conventional symbol-spaced equalizer. The gains at BER $=10^{-3}$ in SNR compared to equalizer with $m=2$ are about $2.1 \mathrm{~dB}$ and $5 \mathrm{~dB}$ for the equalizers with $m=4$ and $m=8$ respectively.

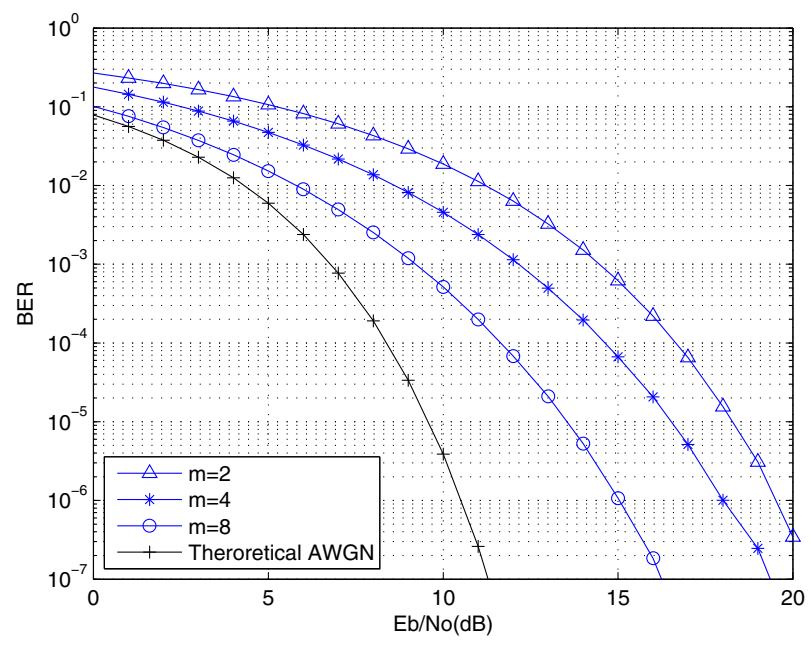

Fig. 8: Performance of fractionally-spaced FDE.

\section{CONCLUSION}

With the aid of the relay and the fractionally-spaced FDE, the performance of the UA communication system is significantly improved compared with the conventional equalizer in non-cooperative communication scheme. In this paper, a two-hop relay system is evaluated by simulation to show that better performance can be achieved in comparison with noncooperative system.

\section{REFERENCES}

[1] D. Lucani, M. Stojanovic, and M. Medard, "On the relationship between transmission power and capacity of an underwater acoustic communication channel," in Proc. MTS/IEEE OCEANS 2008, Apr. 2008, pp. 1-6.

[2] M. Stojanovic, "Recent advances in high-speed underwater acoustic communications," IEEE Journal of Oceanic Engineering, vol. 21, no. 2, pp. 125-136, Apr. 1996.

[3] Z. Feng, K. F. C. Yiu, K. L. Teo, and S. Nordholm, "Design of broadband beamformers with low complexity," EURASIP Journal Advances in Signal Processing, vol. 2012, no. 1, pp. 62-73, 2012.

[4] A. Nasri, R. Schober, and I. Blake, "Performance and optimization of amplify-and-forward cooperative diversity systems in generic noise and interference," IEEE Transactions on Wireless Communications, vol. 10, no. 4, pp. 1132-1143, Apr. 2011.

[5] B. Zhang, Z. He, K. Niu, L. Zhang, "Robust linear beamforming for MIMO relay broadcast channel with limited feedback," IEEE Signal Processing Letters, vol. 17, pp. 209-212, Feb. 2010.

[6] Y. Rong "Multi-hop non-regenerative MIMO relays - QoS considerations," IEEE Trans. Signal Processing, vol. 59, pp. 290-303, Jan. 2011.

[7] B. Li, Q. Wang, G. Lu, Y. Chang, and D. Yang, "Linear MMSE frequency domain equalization with colored noise," in Proc. IEEE VTC 2007 Fall, Oct. 2007, pp. 1152-1156.

[8] P. P. Vaidyanathan and B. Vrcelj, "Theory of fractionally spaced cyclicprefix equalizers," in Proc. IEEE ICASSP 2002, vol. 2, May 2002, pp. $1277-1280$. 